\title{
Human-Animal Chimera: A Neuro Driven Discussion? Comparison of Three Leading European Research Countries
}

\author{
Laura Yenisa Cabrera Trujillo • \\ Sabrina Engel-Glatter
}

Received: 28 February 2014/Accepted: 22 May 2014/Published online: 3 June 2014

(C) Springer Science+Business Media Dordrecht 2014

\begin{abstract}
Research with human-animal chimera raises a number of ethical concerns, especially when neural stem cells are transplanted into the brains of nonhuman primates (NHPs). Besides animal welfare concerns and ethical issues associated with the use of embryonic stem cells, the research is also regarded as controversial from the standpoint of NHPs developing cognitive or behavioural capabilities that are regarded as "unique" to humans. However, scientists are urging to test new therapeutic approaches for neurological diseases in primate models as they better mimic human physiology than all current animal models. As a response, various countries have issued reports on the topic. Our paper summarizes the ethical issues raised by research with human-animal brain chimeras and compares the relevant regulatory instruments and different recommendations issued in national reports from three important European research nations: Germany, Switzerland and the United Kingdom. We assess and discuss the focus and priorities set by the different reports, review various reasons for and perspectives on the importance of the brain in chimera research, and identify critical points in the reports that warrant further specification and debate.
\end{abstract}

Keywords Human-animal chimera $\cdot$ Moral status $\cdot$ Neuroethics $\cdot$ Stem cells

\section{Introduction}

Neurological disorders such as Alzheimer's and Parkinson's disease are severe conditions characterized by progressive nervous system dysfunction. It is estimated that 36 million worldwide suffer from Alzheimer's disease (Alzheimer's Disease

L. Y. Cabrera Trujillo $(\bowtie) \cdot$ S. Engel-Glatter

Institute of Biomedical Ethics, University of Basel, Bernoullistrasse 28, 4056 Basel, Switzerland e-mail: Laura.cabrera@unibas.ch 
International 2009), and 6.3 million people from Parkinson's disease (World Health Organization 2003). It is expected that these conditions will affect more and more people in the future. For most neurological disorders, including Parkinson's and Alzheimer's disease, no curative treatment is available so far.

Considering the high prevalence of these neurological diseases and the burden they cause for society (Boland and Stacy 2012), research efforts aiming to discover new therapeutic approaches have intensified over recent years (European Commission 2013). One major approach to new therapeutics, for example in Parkinson's disease, has been the replacement of affected tissue in patient's brains with graft tissue or stem cells to regain lost functionality (Dunnett and Rosser 2014). Initial clinical trials from the 1980's transplanting human foetal donor tissue into Parkinson's patient brains showed some success (Lindvall et al. 1988). However, due to ethical and quality issues with primary foetal tissues, researchers are now focusing on human stem cell transplantation.

Before moving to human clinical trials, the proof-of-principle and safety of potential new stem cell therapies has to be tested in various animal models. Although animal research remains controversial, policy makers and regulators in the European Union (EU) permit the use of certain animals provided animal welfare protocols are followed. Currently, rodents, such as mice and rats, are the preferred animal model for biomedical research for various reasons: they reproduce quickly, can be genetically manipulated, and are relatively inexpensive. However, it remains unclear how well results from experiments with rodents can be extrapolated to humans. Researchers have argued for the use of closer human relatives as research subjects, especially non-human primates (NHP). According to the scientific community, NHPs present various advantages over smaller animal models including a longer lifespan and similarity of physiological features (Deutscher Ethikrat 2011; Harding et al. 2013) making them better models of human disease.

Several recent studies have paved the way to an increased use of NHPs as animal models for studying cellular replacement therapy. For example, the paper by Emborg and colleagues provided the proof-of-principle that derivatives of induced pluripotent stem cells (iPSCs) can be engrafted and survive in the primate brain (Emborg et al. 2013). Redmond and Bjugstad have shown the safety and feasibility of cell replacement therapies for neurological disorders in NHP models. They have seen functional improvement in African green monkeys with Parkinson's disease symptoms upon transplantation of undifferentiated human neural stem cells (hNSCs) into monkeys' brains (Redmond et al. 2007). Another successful approach has been published by Pluchino and colleagues. Following transplantation of hNSCs into an NHP model approximating the clinical and complex neuropathological situation of human multiple sclerosis (MS), the researchers observed a reduction in severity of the disease, and the symptoms-related mortality (Pluchino et al. 2009). The papers suggest that NHP's are suitable models for studying cellular replacement therapies in neural systems, making it highly likely that research on stem cell therapies will increase in the future.

Furthermore, recent advances in the field of stem cell research, in particular with embryonic stem cell (ESCs) and iPSCs, will most likely impact the use of NHPs for research purposes. These include the ability to reprogram iPSCs directly to neurons 
(Vierbuchen et al. 2010), and to obtain disease-relevant neurons (Park et al. 2008). In addition, ESCs can now be programmed into neuronal cells in the absence of proliferative contaminant cells (Kriks et al. 2011). Another factor is that widespread concerns over adverse events such as immunoreactions associated with the use of tissue derived from iPSCs or ESCs have been eased by recent studies (Araki et al. 2013; Baker 2013). As Parkinson's and Alzheimer's disease are characterized by the loss of specialized neurons, cellular replacement of these by iPSC-derived neurons seems now feasible (Barker 2012).

However, research involving NHPs raises various ethical concerns. These include issues associated with the use of animals that are, from an evolutionary point of view, closely related to humans, and concerns over the potential of NHPs acquiring human capabilities ${ }^{1}$ as a result of human neural stem cell transplantation. Accordingly, we believe it is of vital importance to discuss the ethical concerns associated with human-NHP brain chimera research and find sensible solutions before research intensifies.

\section{Ethical Concerns Associated with the Creation of Human Non-human Chimeras}

The main ethical concerns that human-animal chimera research raises have already been discussed by several scholars. Among these concerns, the following examples are commonly referred to, but not regarded as strong enough to ban this type of research: moral taboo ${ }^{2}$ and tampering with natural law, including the yuck factor as well as religious based arguments ${ }^{3}$ (Human Fertilisation and Embryology Authority 2007b; de Melo-Martin 2009; ESTOOLS 2008; Hunther 2009; Karpowicz et al. 2005; The Danish Council of Ethics 2008); violating the integrity of the species (Karpowicz et al. 2005; The Danish Council of Ethics 2008); and moral confusion ${ }^{4}$ (Robert and Baylis 2003). Other more pragmatic concerns deal with the possibility of "experimental problems of rejection, transfer of viral infections and different physiological environments" (Grunwell et al. 2009).

Another main set of concerns associated with the creation of human non-human chimeras relates to animal welfare (Deutscher Ethikrat 2011; Greely et al. 2007; Hunther 2009; Nuffield Council on Bioethics 2005; Swiss Academy of Medical Sciences 2009; Swiss Academies of Arts and Sciences 2010; The Academy of Medical Sciences 2011). These arguments focus on animal suffering and their

\footnotetext{
${ }^{1}$ By human capabilities we mean capabilities that are regarded to a great extent as uniquely human.

2 According to the moral taboo argument it is sufficient that certain practices elicit repugnance to indicate that they are wrong (c.f. (Karpowicz et al. 2005).

3 Both, the tampering with natural law concern and the "yuck" factor, are in line with the long legacy of human concerns in relation to the creation of "monsters". Well-known fiction characters like the characters from the Island of Dr. Moreau, with "monsters-like" creatures resulting from a mixture of human and animals, are examples of the various public anxieties that human-animal chimera research embodies.

4 According to Robert and Baylis " $[\mathrm{t}]$ he engineering of creatures that are part human and part nonhuman animal is objectionable because the existence of such beings would introduce inexorable moral confusion in our existing relationships with nonhuman animals and in our future relationships with part human hybrids and chimeras" (p. 9). cf. (ESTOOLS 2008).
} 
capacity to feel pain, both physical and psychological, as well as the questionable need and benefit of using animals in research.

A core concern, and focus of this paper, is related to "moral status". This concern includes critique over the use of human embryos in research, as well as the use of animals that are regarded to have a high moral status (e.g. Great Apes ${ }^{5}$ ) for research purposes. A related concern with regards to brain chimera research is that of human dignity. The transplantation of human derived material may create a chimeric organism with cognitive or behavioural human-like capabilities, which could question the culturally- and species-related understanding of human dignity. In the case of research involving NHPs, the concern is even greater due to their close evolutionary relation to humans.

This paper discusses the ethical and regulatory concerns associated with chimera research, especially research aiming to transplant human neural cells into NHP brains. It compares these concerns across three European countries: Germany, Switzerland and the United Kingdom (UK). The three countries were chosen as they are among the leading European nations in biomedical research, and because they present three different perspectives on brain chimera research shaped by the different historical and legal backgrounds of each country.

\section{Human- NHP Brain Chimera Research in the UK, Germany and Switzerland}

\section{United Kingdom: "Animals Containing Human Material”}

The UK has issued a number of reports evaluating ethical issues of chimera research, including the 2005 report "The ethics of research involving animals" published by the Nuffield Council of Ethics (Nuffield Council on Bioethics 2005); the report "Hybrids and chimera: A consultation on the ethical and social implications of creating human/animal embryos in research" issued in 2007 by the Human Fertilisation and Embryology Authority (Human Fertilisation and Embriology Authority 2007a; Human Fertilisation and Embryology Authority 2007b); and the 2011 report written by the Academy of Medical Sciences (AMS) "Animals Containing Human Material" (The Academy of Medical Sciences 2011). The AMS report focuses on research involving "the introduction of human DNA sequence into animals, or the mixing of human and animal cells or tissues" (The Academy of Medical Sciences 2011, 5). The latter report is the most recent report issued by a UK body and aims to be a framework for governing ethically sensitive research in this area as well as an example for other countries to follow. As a result, we will mainly focus on this report in the discussion below.

The AMS report recommends more international awareness, consistency of research practices, and the development of international guidelines and standards. As part of the report a public consultation was conducted which found a high degree of public acceptance for this type of research provided the research is "well

\footnotetext{
${ }^{5}$ Great apes include the two chimpanzees, the common chimpanzee, and the bonobo, as well as the orangutan and gorilla.
} 
regulated and justified" (Academy of Medical Sciences 2010). However, it was also found that the public regards research involving the brain, reproductive tissue and aspects of human-like appearance as problematic. The report emphasizes that these issues need to be discussed and addressed by the research community, regulatory bodies, and the public at large.

\section{Germany: Human-Animal Mixtures in Research}

In Germany, the Ethics Council published an opinion paper in September 2011 addressing human-animal mixtures in research (Deutscher Ethikrat 2011). The paper, which focuses on the transfer of human material to animals, intends to clarify the "distinction between humans and animals, to facilitate the evaluation of developments with ethical implications in research involving the creation of human-animal mixtures, and to indicate where action is called for on the part of science, society or politics" [p. 10]. To our knowledge this has been the only document addressing chimera research published by a relevant German body.

\section{Switzerland: Interspecies Crosses and Aspects of Animal Protection}

Two years before publication of the German report, the Ethics Committee for Animal Studies of the Swiss Academy of Medical Sciences (SAMS) and the Swiss Academy of Sciences (SAS) had published a position statement paper (Swiss Academy of Medical Sciences 2009) on interspecies crosses. The paper does not explicitly refer to brain chimeras, focusing primarily on aspects of animal protection. Another relevant report was published in 2006 by the Swiss National Advisory Commission on Biomedical Ethics (NEK 2006) "Research involving human embryos and foetuses", which discusses human ethical aspects of chimera research. Given that the focus of this paper is human-animal chimeras, we will primarily consider the 2009 position statement paper on interspecies crosses.

The various documents mentioned above review ethical as well as regulatory issues of interspecies research. The AMS's report from the UK, as well as the German report, focuses on the use of human material in animals. In contrast, the Swiss position paper discusses more general issues with no particular focus on transplantation of human material to animals. All reports acknowledge the use of stem cell transplantation as one of the main approaches for creating human-NHP chimeras. Special consideration is dedicated to the brain and reproductive system. In fact, little regard is paid to other organs.

In addition, except in the case of Switzerland, the protection and dignity of humans, and, to a lesser extent, the protection and dignity of the animals are a main focus of the reports. Likewise, it can be said that while the three reports do acknowledge the sentience of animals, only the Swiss report seems to take increased attention in relation to the interests of animals and not just those of humans. However, none of the reports sets the focus on the suffering of animals.

In this section, we will compare the similarities and differences of the three reports regarding relevant ethical concerns associated with human-NHP brain chimera research. 


\section{Ethical and Social Concerns}

The various risks associated with creating human-animal chimera are one main focus of the reports. For example, all reports discuss health risks for current and future patients, including the risk of transferring animal-specific infectious diseases to humans. However, these are not seen as unique to chimera research or as insuperable. Issues beyond human health are addressed by both the UK and the German reports. The UK report addresses social concerns on how culture and science fiction shape the public imagery of interspecies chimera research, creating hype and wrong ideas of what is scientifically possible to date, as well as environmental risks. The latter involves the possibility that these creatures will be released or escape from laboratory settings and put a threat to current ecosystems (Table 1).

The value of research for society is highlighted in all main documents. It is seen as an important goal to preserve freedom of research. For instance, the Swiss National Ethics committee argues that "research should therefore only be restricted by society for good reasons. Society may also benefit from this freedom, since it can never be known in advance what findings of basic research may yield beneficial applications" (NEK 2006). In case of the UK, the benefits of research are acknowledged as part of a "social responsibility to facilitate medical research", and are seen as to be in conflict with the responsibilities humans have towards animals (The Academy of Medical Sciences 2011).

Even though the documents emphasize the importance of animal models for advancing research and improving human health, there is also support for certain restrictions in relation to animal experimentation (See Table 2). For example, the German report argues that "the less important the purpose of the experiment, the more weight is attached to the animal's welfare" (Deutscher Ethikrat 2011).

In general, animal suffering is a main concern of the various reports. All reports cover the issue of animal husbandry and quality of living as part of their animal welfare concerns. However, the 3R's approach-reduce, refine, replace-introduced in the 1950s to minimize animal pain and suffering, is only explicitly acknowledged by the UK report. ${ }^{6}$ The German and the UK reports particularly emphasize that the use of primates deserves special considerations. In particular, the argument stated by these reports is that given their closeness to humans, the research goals have to be of special and high value to justify research with primates.

One aspect, which is regarded as being of high relevance especially in the case of primate chimera research, is the possibility of psychological effects for the animal as a result of transplanting human neurons into its brain. The UK states that "an animal might in some way be made more 'self-aware' and therefore capable of experiencing a greater degree of suffering" (The Academy of Medical Sciences 2011). The Swiss report refers to article 25 in the Swiss Animal Protection Ordinance which prohibits the "breeding animals with deviations from species-

\footnotetext{
6 The 3R's entail to refine the procedures applied to animals so they do not suffer, reduce the number of animals use as well as replace higher animals with insentient material (Russell and Burch 1959).
} 
Table 1 Medical/clinical/scientific research ethics ethical considerations

\begin{tabular}{llll}
\hline Ethical consideration & Germany & Switzerland & UK \\
\hline Risks to humans (e.g. new infectious diseases) & $\boldsymbol{V}$ & $\checkmark$ & $\checkmark$ \\
Social disruption and/or environmental risks & $\boldsymbol{V}$ & & \\
Value of research & $\boldsymbol{V}$ & $\boldsymbol{V}$ & $\boldsymbol{V}$ \\
Freedom of research & $\boldsymbol{V}$ & $\boldsymbol{V}$ & $\boldsymbol{V}$ \\
Human dignity concerns & $\boldsymbol{V}$ & $\boldsymbol{V}$ & \\
\hline
\end{tabular}

Table 2 Animal Welfare Ethical Considerations

\begin{tabular}{llcc}
\hline Ethical consideration & Germany & Switzerland & UK \\
\hline Pain and suffering & $\checkmark$ & $\checkmark$ & $\checkmark$ \\
Instrumentalisation/adulteration of life & $\checkmark$ & $\checkmark$ & $\checkmark$ \\
Quality of life and species integrity & $\checkmark$ & $\checkmark$ & $\checkmark$ \\
Moral status & $\checkmark$ & $\checkmark$ & $\checkmark$ \\
Symbolic meaning & $\boldsymbol{V}$ & & $\boldsymbol{V}$ \\
\hline
\end{tabular}

typical behaviour that could make cohabitation with members of the same species very difficult or impossible".?

The instrumentalisation of life is another main concern covered by the reports. It is highlighted that research with animals should only be allowed if the possible benefits that the research claims to bring couldn't be achieved by any other means, and as long as animals are treated as entities with their own set of species-specific interest. The German report refers not only to the instrumentalisation of animals but also of humans. The UK report does not explicitly mention this concern. However, it does cover the stewardship of humans and their responsibilities towards animals.

Mixing two species by transplanting human neuronal material into animal brains may also cause concern with respect to species-specific integrity and the transgression of 'natural' species boundaries. Although many scholars have argued that the concept of a species is ambivalent and does not really enable us to reach strong arguments about whether interspecies research should be carried out or not (Greene et al. 2005; Hunther 2009; Karpowicz et al. 2005; Robert and Baylis 2003), it is nevertheless mentioned in the reports. For instance, the UK report points out that "the process of humanizing an animal undermines the integrity of the animal's inherent life-form" (The Academy of Medical Sciences 2011). However, it also concludes that chimera research focused on advancing medical research "does not bring significant new ethical problems as compared with other kinds of medical research which use animals". The German report also acknowledges that transgressing species boundaries may lead to problems with species categorization

\footnotetext{
${ }^{7}$ While animal suffering and sentience was a main concern in all reports, it is interesting, as one of our reviewers brought to our attention, that animal feelings are not a main focus. This could be to a great extent due to the anthropological-bias, in which human interests are prioritized over animal interests. More research is needed in this area to better understand the reasons at play.
} 
and hence to difficulties with assigning a moral status to the chimera. While the report concludes that neither man's duty to preserve the natural species on earth, nor the 'unnaturalness' of creating human-animal chimera ('Playing god' argument), are sound arguments against this kind of research, it considers relevant for an ethical discussion whether manipulation of the animal has "repercussions on capabilities relevant to an organism's moral status" (Deutscher Ethikrat 2011), such as humanlike cognitive capabilities.

All reports address concerns in relation to dignity and moral status. The UK and German reports focus on difficulties with assigning a moral status to human-animal chimera, and the possibility of violating human dignity by creating such chimera, whereas Switzerland in its 2009 position paper focuses on issues connected to animal dignity. The reports discuss that manipulations instantiating human like cognitive or behavioural capabilities, such as consciousness or sentience, would be ethically problematic. The German and Swiss reports both highlight that human dignity is at stake both at the individual level and at the human species level. ${ }^{8}$ In contrast, the UK report only considers the latter in its AMS report.

Finally, the UK and German reports discuss the symbolic significance of the distinction between humans and animals. In particular, the reports highlight the special status of humans, which is granted due to certain capacities "not present" in animals, such as rationality and self-awareness. This symbolic concern highlights the idea of the supremacy that is given to be human, at times explicitly (when certain capacities are regarded as unique to humans) and at times implicitly (for instance when animal concerns are overridden by benefits to humans).

\section{Summary of Recommendations}

The documents analysed use a mixture of utilitarian and deontological perspectives. The main recommendations are summarized in Table 3.

All reports share the view that cellular replacement therapy and chimera research are important. The recommendations support this type of research provided the research goals are valid and animal welfare concerns are taken into consideration. None of the reports regards the species boundary as being universal and categorical inviolable. Hence, no recommendation relies on this argument for regulating human-NHP chimeras. However, given the closeness of primates to humans, all countries take into account the possibility that human characteristics may emerge. As a result, they call for extra oversight or for more research with other animals before proceeding with such research in NHPs. The reports also re-emphasize the agreement on banning such research with Great Apes, hence supporting the current approach in all three countries to not conduct any kind of research with Great Apes.

The different recommendations point to the underlying conflict surrounding human-NHP chimeras. Human benefits are assessed from a utilitarian perspective (e.g. better therapies for humans), whereas most objections are deontological in nature (e.g. protection of human dignity or the protection of animal interests)

\footnotetext{
8 "The combining of human and animal can harm not only the dignity of humanity but also the human dignity and personality of the unique individual human being" (Reusser and Schweizer 2008).
} 
Table 3 Recommendations from the different reports, relevant for human-NHP chimeras

\begin{tabular}{|c|c|}
\hline \multirow[t]{3}{*}{ Germany (Deutscher Ethikrat 2011) } & $\begin{array}{l}\text { IV.1 The creation of brain chimeras by the transfer of human } \\
\text { cells to mammals other than primates is ethically acceptable if: }\end{array}$ \\
\hline & $\begin{array}{l}\text { 1) the objective of the research is of overriding importance, } \\
\text { especially in terms of the expected medical benefit to humanity; }\end{array}$ \\
\hline & $\begin{array}{l}\text { 2) the generally applicable ethical requirements of animal welfare } \\
\text { are satisfied; }\end{array}$ \\
\hline
\end{tabular}

Switzerland (Swiss Academy of Medical Sciences 2009; NEK 2006)

UK (The Academy of Medical Sciences 2011)
To ensure that the conditions under which the animal is kept are appropriate to its species, the degree of cell integration and the behaviour of the animal after birth should preferably be monitored.

IV.2 The insertion of brain-specific human cells into primate brains should be permissible only after an interdisciplinary evaluation process

IV.3 Research involving the introduction of brain-specific human cells into the brains of great apes should not be carried out

The provisions of the animal protection legislation must be strictly observed and the issues relating to the respect of the dignity of the animal must be clarified in each individual case

- It must be assessed with particular care whether the consequences that the introduction of numerous cells from other animal species or humans can have for the well-being of the generated animals and whether the latter's physiological and ethological needs can be adequately met

NEK opinion no11. Section 3.3.4 specially on chimera and hybrids

NEK-CNE (National Ethics Commission for Human Medicine) is inclined to accommodate a gradualist perspective in the ethical assessment, taking into consideration the species involved and stage of development

For a majority of the Commission, it appears to be a more convincing strategy to prohibit the production of chimeras resulting from the combination of human stem cells with animal embryos than to seek to define limits

It is proposed three categories into which experiments involving animals containing human material can be classified. The type of chimera research discussed here falls into category 2 and 3

Category 2. Experiments that are likely to result in [substantial modification of an animal's brain that may make the brain function potentially more 'human-like', particularly in large animals] should be scrutinized by a national expert multidisciplinary body that also advises on more general aspects

Category 3. A very limited number of studies should not currently be undertaken because they raise very strong ethical concerns or lack sufficient scientific justification, such as: Transplantation of sufficient human-derived neural cells into an NHP as to make it possible, in the judgement of the national expert body, that there could be substantial functional modification of the NHP brain, such as to engender 'humanlike' behaviour 
(Magnani 1999). The UK report showed a more gradualist perspective in terms of moral status, whereas the German and Swiss reports focused on the intrinsic moral status of animals. ${ }^{9}$ The German report, in particular, refers to the precautionary principle "as a basis for addressing developments in research on human-animal mixtures". In the recommendation part, the UK report argues in particular for sufficient flexibility in order to be able to evolve with advances of scientific knowledge and changes of social attitudes. The Swiss recommendations reaffirm the dignity of the animal, which could be seen as a more restrictive perspective compared to the other two countries. However, the balance between freedom of research and innovation, two main pillars of the different regulations in Switzerland, might translate to a similar degree of restrictiveness with regards to Germany.

\section{Legal and Regulatory Considerations}

Currently, no specific regulations or laws addressing human-animal chimera research in Germany, Switzerland or the UK are in place. However, there are some international and national legal instruments relevant for chimera research, which we will briefly discuss in the next section.

\section{International Scope}

Report on ethical standards for human-to-animal chimera experiments in stem cell research (ISSCR) (Hyun et al. 2007): These non-binding guidelines intended for the international research community are the only international recommendations explicitly addressing chimera research. The guidelines call for more monitoring of this type of experiments (Rec. 3 and 4) asking investigators and institutions to make the appropriate adjustments to research protocols in order to deal with new data or unanticipated responses from animal subjects including "novel signals demonstrating deterioration or enhancement of an animal's condition and other factors pertinent to withdraw the animal from the study" (Rec. 6). Finally, the guidelines recommend that research with the potential to "create humanized cognition, awareness, or other mental attributes, while not absolutely prohibited, should be subject to close scrutiny, taking the most careful steps to collect data pertinent to ethical protection of animal subjects, and an extraordinary degree of justification".

\section{European Scope}

EU Directive On the protection of animals used for scientific purposes (Directive 2010/63/EU): This directive updates and replaces the 1986 Directive 86/609/EEC on animal experimentation, with the aim to improve animal welfare standards across Europe and to harmonize them between EU countries. Even though the directive only establishes minimum standards, it enables its member states to retain flexibility

\footnotetext{
${ }^{9}$ However, the Swiss recommendation (NEK) discusses the plausibility of taking such a gradualist approach.
} 
in order to maintain national rules aimed at more extensive protection of animals. It focuses primarily on three issues: experiments on primates, experiments on Great Apes and experiments which cause severe pain. The directive also extends protection to foetal forms of mammals used for scientific or educational purposes (art. 1). While Switzerland has no legal obligation to transpose EU directives, in the particular case of animal protection, it has a more rigorous legislation on protecting animals than those set by the EU Directive.

While the directive intends to improve animal welfare, some scientists argue that the revision of the directive will fundamentally affect basic research as well as medical research, especially macaque based research ${ }^{10}$ (Suran and Wolinsky 2009). It has also been argued that it would represent yet another bureaucratic burden (Ricceri and Vitale 2011), and that it could lead to skewed conclusions of pharmacological and behavioural studies (Macrì et al. 2013). ${ }^{11}$

\section{National Scope}

Germany and Switzerland have statutes within their constitutions applicable to interspecies research. In contrast, the UK does not have a single constitutional document but rather various written documents, such as statutes and treaties, that form the British constitution. Some of them apply to chimera research (see Table 4 for a summary).

Besides the generally applicable fundamental rights such as human dignity, and freedom of research, the German and Swiss constitution include other articles relevant to chimera research. The German Basic Law refers to the protection of animals (art. 20a), however, there is no specific provision referring to human-animal mixtures. The Swiss constitution outlines in art. 119(2b) that "non-human reproductive and genetic material may neither be introduced into nor combined with human reproductive material". A commentary on this article was published in 2008 called the St. Gallen Commentary on the Federal Constitution, stating that "the provision also prohibits the formation of so-called inter-species hybrids (mongrels) through the fusion of the gametes of different species, and of interspecies chimeras" (Reusser and Schweizer 2008).

The Swiss constitution furthermore promotes scientific research and innovation (art. 64), and includes provisions for gene technology in the non-human field that may represent a threat to the dignity of living beings (art. 120). Finally, in 2004, the Swiss constitution was changed in order to acknowledge the "dignity of living

\footnotetext{
10 Since 2004, the Swiss constitution includes the protection of the dignity of all living beings, including animals and plants (Article 120 Paragraph). As a result, a court in 2009 rejected two neurological projects using macaque monkeys (Menache 2010; Suran and Wolinsky 2009).

11 The EU Directive requires environmental enrichment, in which the environment animals are kept in "shall be adapted to the species and individual needs". However, these environmental modifications have been found to affect a number of observations measured in classical pharmacological and behavioural protocols, which could skew conclusions using these new environments compared to classical animal facility rearing.
} 


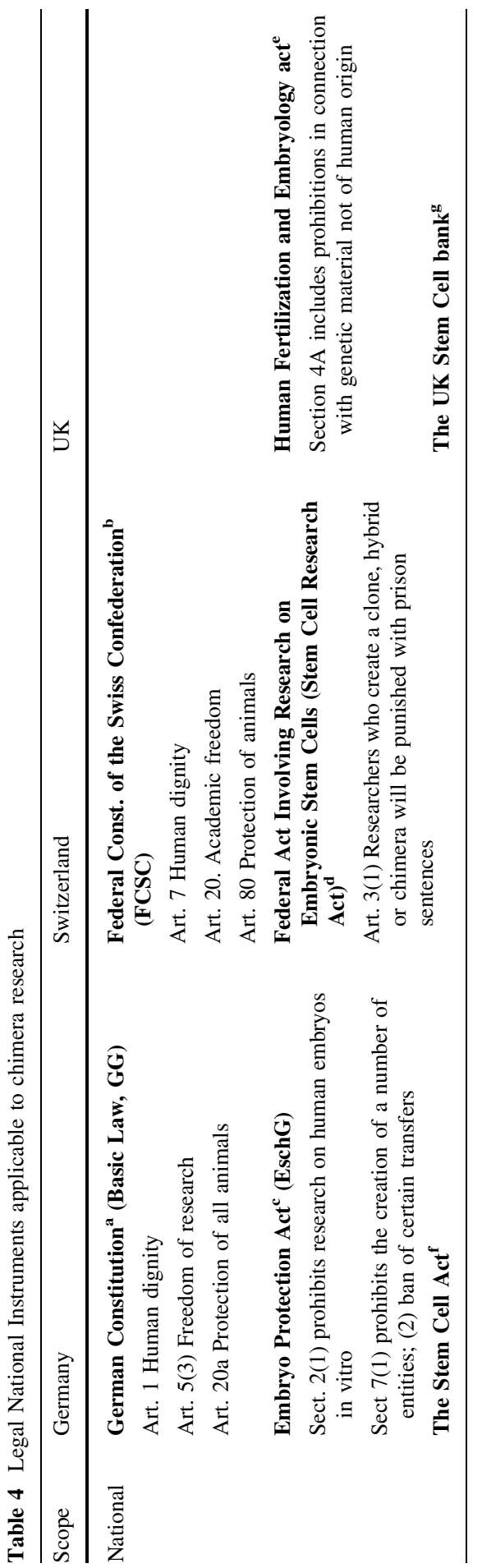




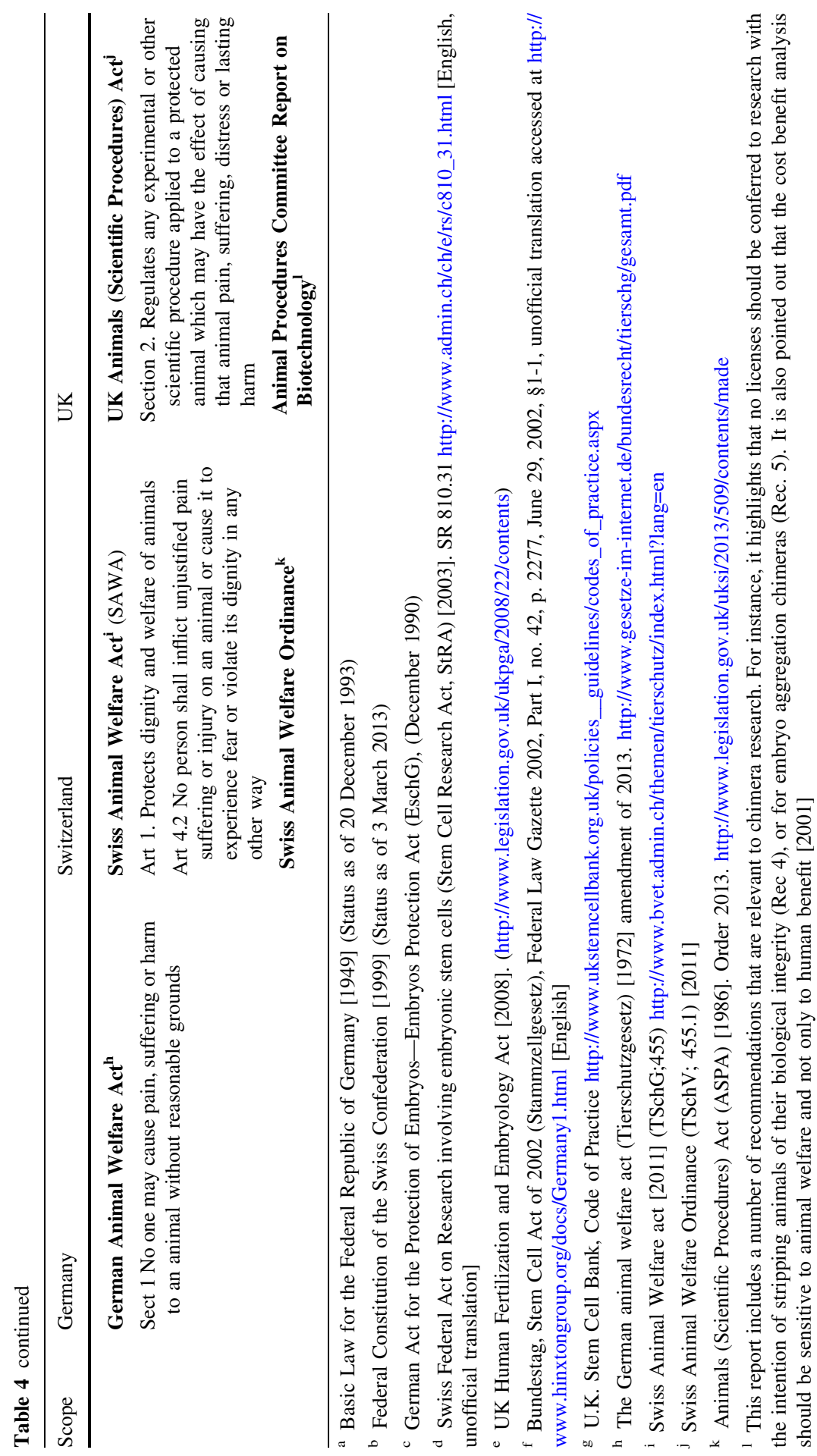


beings" (art. 80), including animals and plants. ${ }^{12}$ This last article differentiates the Swiss regulation from German and British regulation and makes Switzerland the European country with the strictest animal protection regulation, extending even beyond what is required by the new European directive. ${ }^{13}$

In summary, comparing the different regulations regarding animal protection, the Swiss legislation is more restrictive than the German legislation. In contrast, the UK legislation is more liberal, which is reflected by the fact that the generation of chimeras for research is permitted. ${ }^{14}$ Because no legislation covers the generation of human-animal chimera in Germany, the ethics council has urged German policy makers to clarify the situation.

\section{Research with Human Embryos}

The creation of human-animal mixtures may imply the use of human or animal embryos. Hence, regulations addressing embryonic research issued by the three countries may apply to human-animal chimera research. While the German Embryo Protection Act (ESchG) does not explicitly address the problem of human-animal mixtures, it can be interpreted to include embryos that are not "purely" human. However, it would only apply to such embryos made through gamete fusion, not through somatic nuclei transfer. The German regulation bans any manipulation of human embryos, except if the purpose is to preserve the embryo. In contrast, the Swiss Federal Act on research on embryonic stem cell explicitly prohibits the creation of chimera (art. 3). ${ }^{15}$ The UK legislation, in form of the Human Fertilization and Embryology Act, is more flexible as it states that chimera research is not prohibited, albeit being strictly regulated.

\section{Stem Cell Research}

Regulations addressing stem cell research are also relevant for human-animal chimera research. All three countries have issued specific regulations addressing stem cells. However, they do not explicitly involve human-animal research. In

\footnotetext{
${ }^{12}$ Using the term dignity in relation to animals has been questioned. The position paper issued by the Swiss Academies of Arts and Sciences on "the dignity of animals and the evaluation of interests in the Swiss Animal Protection Act" (Swiss Academies of Arts and Sciences 2010) draws attention to a lack of consistency in the use of terminology referring to dignity of animals in the Swiss Federal Constitution, the animal Protection Act and the animal Protection Ordinance. Moreover, in practical terms it is not clear whether the term "dignity" as used under Swiss regulation provides enhanced protection compared to any of the other two countries.

${ }^{13}$ While the German animal welfare regulation does not mention dignity it does mention that animals have rights.

14 "In general, the creation of all types of human-animal chimera or hybrid embryos should be allowed for research purposes under licence by the regulator" (Human Fertilisation and Embriology Authority 2007a).

15 They refer to chimera formation as the fusion of totipotent cells from two or more genetically different embryos. This definition of chimera does not cover secondary chimeras, in which cell grafting takes place at a later developmental stage. The same statute is found in the Swiss Federal act on reproductive medicine under art. 36.
} 
Germany, the Stem Cell Act prioritizes adult stem cells but permits, under strict conditions, the importation of embryonic stem cells generated before 01.05.2007. In Switzerland, embryonic stem cell lines can be imported specifically for research purposes under strict licensing conditions, and stem cells can be derived from surplus IVF embryos (up to 7 days old). However, in reality stem cell lines cannot be produced in Switzerland, as the generation of surplus embryos is currently not permitted. ${ }^{16}$ The regulatory framework of the UK aims to cover all forms of stem cell research while balancing the need for governance and commercial development. It is governed by a 'Code of Practice', which aims to provide guidance and assistance on best practice to those working with stem cell lines in the UK. While the code does refer to chimera research, it only applies in cases where animal material is mixed with humans, and in which the human component is dominant.

Animal Regulation

In all three countries, licenses and ethics committee approval are essential for those conducting experiments with animals. ${ }^{17}$ Moreover, all current animal regulation emphasizes the point that no one should cause unjustified pain, suffering or harm to animals. Germany and the UK will have to adapt their national regulations in order to follow the new EU directive 2010/63 on animal experimentation mentioned above.

\section{A Neuro Driven Discussion?}

The progress in cellular replacement therapy and its associated ethical concerns have caught the attention of ethics commissions and regulators across Europe. Particularly, the human brain is a main cause for ethical concern. This is true also beyond Europe. For example, the US National Academy of Sciences states: "Perhaps no organ that could be exposed to hES cells raises more sensitive questions than the animal brain, whose biochemistry or architecture might be affected by the presence of human cells" (The National Academies 2005, 41).

One may ask why the brain receives so much attention. First of all, most modern Western societies are neurocentric societies. That is to say, the discussion involving the brain has taken dominance over other topics. This trend is reflected by the emergence of many fields in which neuroscience has a key role: neurolaw, neuroethics, neuroeconomics, neuroeducation, neurofinance, neuromarketing. Scholars such as Nikolas Rose even argue that we have turned ourselves into neurochemical selves (Rose 2009). In addition to this, since ancient times the brain has played a key role. For instance, for Hippocrates, the brain was the messenger and the interpreter of consciousness, as well as the most important human organ.

\footnotetext{
16 Switzerland is planning to change the Federal Constitution (Bundesverfassung) and the reproduction medicine law (Fortpflanzungsmedizingesetz) to allow preimplantation genetic diagnosis. The current draft laws include sections that would allow the generation of surplus embryos and cryopreservation.

17 In the UK, there are certain interventions that do not require a license (art. 2.34) (Secretary of State for the Home Department 1986). .
} 
The brain has also been considered as the seat of our self-identity, autonomy and self-narrative (Fangerau et al. 2011; Greely et al. 2007; Grunwell et al. 2009). Furthermore, the brain is considered to be the bodily "centre of control", and the organ in charge of affective and cognitive capacities of reasoning and decisionmaking. It is from all these different considerations that the brain has been regarded as the most important human organ.

With regards to brain chimera research a main concern is that animals may develop features that have been regarded as uniquely human. Robert Streiffer has argued that there can be at least three possible justifications for this concern (Streiffer 2005). One is the claim that this type of research would enable animals to have experiences that we recognize as being unique to humans; the second possible position is that animals might develop human-like cognitive capacities (de MeloMartin 2009; Greely et al. 2007; The Danish Council of Ethics 2008). ${ }^{18}$ Finally a third justification states that given animals, which are generally regarded has having a lower moral status in comparison to humans, will develop capacities and features that would confer them a higher moral status (Deutscher Ethikrat 2011; Greene 2005; Harvey and Salter 2012; Nuffield Council on Bioethics 2005; Robert 2006; Streiffer 2010). Streiffer argues, for instance, that "it would be unacceptable to perform the kinds of invasive, painful, and lethal experiments allowed by animal research regulations on an individual that is, ex hypothesi, so morally similar to a normally functioning human adult that it is on the border of having full moral status" (Streiffer 2010).

Many of the arguments in the reports are in alignment with the last two justifications: animals might develop human-like cognitive abilities, and thus may deserve to be granted a higher moral status. At times they are intertwined. The reports, especially the German report, connect the debate about assigning a moral status to chimera with human capabilities with a possible violation of human dignity. However, making use of the term dignity may result in confusion as it is not clear whose dignity is threatened by the creation of this type of chimeric entities (de Melo-Martin 2009; Hyun et al. 2007).

NHPs are a main focus of ethical concern with regards to human-animal brain chimera. Not only because many reports and papers share the view that a close relationship to humans is an important consideration for assessing the ethical concerns of interspecies brain chimeras (Deutscher Ethikrat 2011; Greene 2005; Nuffield Council on Bioethics 2005; The Academy of Medical Sciences 2011). It is also because many scientists (Grunwell et al. 2009; Kobayashi 2003), and even some members of the general public (The Academy of Medical Sciences 2011), do not expect other animals with human neurons, for example a mouse, to ever develop the type of characteristics that we regard as the basis of moral status. For instance, Karpowics and colleagues argue that given their smaller skull size, shorter gestation period and nonhuman environment, human cells transplanted into mice brains would not "be able to achieve human brain size and [would lack] the human brain

\footnotetext{
${ }^{18}$ Results from Balaban offered proof that complex behaviours could be transferred across closely related species by showing that transfer of various regions of the neural tube from embryonic quails into developing chicken resulted in the transfer of certain aspects of species-typical crowing behaviour (Balaban et al. 1988). .
} 
organization needed to give rise to human neural functions and behaviours" (Karpowicz et al. 2005).

The fact that human functions and behaviours are regarded as essential to human dignity and/or moral status could be contested, as Bayles and Fenton have argued. Not all relevant mental properties associated with human moral worth are unique to humans (Baylis and Fenton 2007). The set of human functions and capacities that are generally considered the basis of moral status are partly what some scholars have referred to as personhood based capacities, including episodic memory, planning, numerosity, theory of mind and social cognition. Great Apes are a clear example of non-human animals with which we share many of these capacities and thus have been protected under stricter regulation than any other primates. ${ }^{19}$

Given that chimera research with NHPs might result in the emergence of features associated with moral status, such research raises ethical concern. Hence, when conducting chimera experiments, the German and UK reports highlight the importance of assessing the developmental stage in which the chimera is being produced, as well as the number and potency of cells involved. Both could impact the features of the resulting chimera (Deutscher Ethikrat 2011; The Academy of Medical Sciences 2011). ${ }^{20}$ Furthermore, two main difficulties are acknowledged by the German report in terms of brain chimera research involving primates. First, the fact that the typical characteristics of the brain normally develop only after birth, so that they cannot be detected in mixed entities prenatally in real time; and, second, that it is in any case "difficult to assign individual patterns of behaviour clearly and unequivocally to the human or animal category" (Deutscher Ethikrat 2011). Similarly, the UK report argues that if a NHP is modified to come to approximate the cognitive capacity of a Great Ape, then it would no longer be deemed appropriate for use in experimentation, given that research on Great Apes is not currently permitted in the UK (The Academy of Medical Sciences 2011).

However, as Nao Kobayashi argues (Kobayashi 2003), the brain could just be seen as another organ composed of different cells. This view is supported by current state of research showing that only a fraction of injected human cells survive, and only a few human marker proteins for neurons and glial cell are expressed several months after transplantation (Tamaki et al. 2002; Uchida et al. 2000). Underlying this type of argumentation is the idea that if we just talk about brain parts, then concerns about moral status and development of cognitive capacities do not arise as these can only arise when we talk about the brain as a whole.

On the basis of this discussion it has been suggested that human dignity based arguments are flawed. Human dignity is not a property of human cells; rather it is a property of human beings (Baylis and Fenton 2007). Similarly, even if a primate brain will predominantly contain human neurons and other brain cells, it does not follow that it will present human-like cognitive abilities. Nurture and the environment also play an important role for the development of cognitive abilities.

\footnotetext{
19 That is not to say that other primates or other non-human animals do not share to some degree these capacities rather that it has been more difficult to assess the extent to which these characteristics are shared.

20 These recommendations are also in line with the ISSCR recommendations.
} 
For example, children who have been raised until the age of seven under conditions of almost total language deprivation, despite intense subsequent training, never learn more than a rudimentary level of communication (Purves et al. 2001). Thus, the potential to develop language is not sufficient for language development.

Correspondingly, who we are (or will become) is significantly shaped by the embodied, embedded and enacted nature of cognition (Held 2006; Walter 2010). ${ }^{21}$ Taking into consideration the importance of the environment, the human bodily functions and nurture for human cognition, one could argue that it is unlikely for human-NHP chimeras to develop human-like capacities. Especially as most of the created entities are, as required by the research protocols, killed after experimentation, and thus will most likely not live long enough to develop such capacities.

On the other hand, humans (and to some extent embryos) are protected based on their potential ${ }^{22}$ to develop human-like capacities, even if they never manage to develop them. One could thus argue that the creation of a chimeric embryo with an inherent potential to develop human-like cognitive abilities, and knowing that it would not be permitted to develop this potentiality, can be regarded as an impermissible harm. Following this line of argumentation, the intrinsic potentiality to develop such actualisable capacities is sufficient for a NHP chimera, already in its embryonic state, to be considered, to use Aristotle's succinct and apt label, a "rational animal" with moral status.

Hence, irrespective of whether human-NHP chimeric embryos are actually allowed to develop beyond birth into an animal with human-like cognitive abilities, the creation of such an embryo alone would be sufficient to challenge our views and raise ethical concerns. Such chimera (in their embryonic stage, respectively) may need to be assigned a higher moral status and be protected by regulations and laws (MacKellar 2013). The same would be true for chimera and chimeric embryos with cognitive abilities equal to those of Great Apes, since based on their abilities these animals are already granted special moral consideration and thus are under more strict protection.

However, it will be impossible to know already during embryonic development whether the created human-NHP chimeric embryo possesses human-like cognitive capacities. And even if such an embryo would be allowed to develop beyond birth, it would be more than challenging to assess the cognitive capacities of the born animal, as our understanding of NHP cognitive capacities is limited and data is difficult to obtain due to methodological challenges of experimental research in primate cognition (Tomasello and Call 2011). It may thus be difficult to interpret whether a given behaviour is outside of the normal range, especially since NHPs already possess some traits of personhood, and how this may impact its moral status.

In this regard, human-NHP chimera research is likely to challenge common views about the special status given to humans and to the human brain. In the future, this may impact ethical and legal considerations of human-animal chimera research. Based on our analysis of the different reports, it can be pointed out that the current regulatory structures seem to downplay the abilities of non-human animals to feel

\footnotetext{
21 These are the main theses of situated cognition theories.

22 This potential does not refer to the individual potential but rather to the species potential.
} 
and reason. Thus, an even more pervasive shift in thinking and prioritizing will have to occur before we can see important and needed changes in this area of research.

\section{Conclusions}

In this paper we summarized and compared the various ethical issues raised by human-NHP chimera research. We also compared the relevant regulatory instruments and different recommendations addressing chimera research issued in national reports from three important European research nations: Germany, Switzerland and the UK. These countries were chosen as they are among the European nations with a strong biomedical research agenda, and because they represent different positions regarding ethical issues raised by new technologies.

In terms of regulation, there are currently national, European and international guidelines and recommendations that are relevant to chimera research. However, there is no specific regulation addressing human-NHP chimera, except for the ISSCR guidelines. In this regard, it might be relevant to graft national and international policies specific to this type of research in order to avoid regulatory gaps, inconsistencies and overlaps. In particular, as research conducted beyond the European landscape, such as in Japan or China, might not be restricted by the same ethical and legal concerns.

The reports discuss a wide range of ethical concerns, especially the German and UK report. The UK presents a more pragmatic approach in terms of dealing with ethical concerns, avoiding its assessment to be based on claims connected with dignity. Instead terms such as human stewardship and responsibilities are used. This does not apply to the German and Swiss reports, as they emphasize the importance of human dignity and moral concerns or, in the Swiss case, animal dignity. There are also commonalities, for example, freedom of research is regarded as an essential part on the scientific agenda of the three countries profiled here. Animal welfare concerns are also intensely discussed. One main concern considered by all reports, and the one that we believe to be of high relevance for guiding human-animal chimera research and its ethical discussion, is the one related to capabilities relevant to an organism moral status.

Overall, our findings suggest that the Swiss legislation could be regarded as the most conservative, in particular, because Switzerland is the only country with a regulatory document prohibiting the creation of chimeras. Furthermore, the Swiss constitution contains an article on the dignity of living beings (art. 120), which has already caused a more restrictive practice with regards to the use of animals, particularly primates, in research. In contrast to Switzerland, the German legislation may be regarded as more liberal as it acknowledges animal rights but does not go as far as to refer to animal dignity. Finally, compared to Germany and Switzerland, the UK follows a more open regulatory approach with regards to chimera research as certain interspecies interventions are already permitted.

In summary, the increasing tension between researchers aiming to move to larger animal models that better mimic human physiology, especially NHPs with the hope to bring new therapy paths, and regulation aiming to grant a more inclusive 
protection of animal's interests, in particular those that are closely related to humans, was reflected in the reports. Thus, it seems that at least in the European context NHPs interest might triumph over the possible promises that primate research could bring for medical therapies. The different issues discussed in the UK and German reports were informed by a national public consultation on the topic of interspecies research. They aimed at fostering a balanced and informed public discussion in order to avoid unsustained fear or concerns that could damage the promises this research has for health and wellbeing. Accordingly, it is to be appreciated that the three countries profiled here have started to get involved in this discussion.

However, while the reports are a good starting point, they lack specific guidance on how to assess whether certain experiments lead to "substantial modification" which engenders "human-like" behaviour. For example the UK report suggests the promotion of a regulation that is gradual, recommending the classification of experiments involving animals containing human material into three categories (see Table 3). Yet, the report does not further specify which experiments should be sorted into this category than what is presented in Table 3. In contrast to the UK and German reports, the majority of the Swiss Commission acknowledged the difficulties with drawing a line and hence supports to prohibit the production of chimeras resulting from the combination of human stem cells with any animal embryo (NEK 2006).

We agree with the UK report that a gradual perspective may provide the needed flexibility to balance freedom of research and ethical concerns, but share the concern of Greene et al. regarding chimera research (Greene et al. 2005): Engrafting higher proportions of human (neuronal) stem cells into NHP embryos, especially in early developmental phases, increases the prospect of more humanlike neural function. Given the different ethical concerns involved and the difficulties with assessing the moral capacities of NHPs, one option may be to prohibit chimera research involving NHP embryos. In contrast, in the light of the current state of research, implantation of human neural cells into adult NHP brains seems to have a lesser impact on the brains function and potential and thus may carry a lesser risk of creating human-NHP chimera with human-like cognitive potential (Greene et al. 2005).

Both, the UK and the German report, suggest installing a national (multidisciplinary) expert body in order to determine the risk associated with certain types of chimera research. While this approach provides flexibility and involves expert knowledge, it remains to be seen whether the discussions and final decision of such a body would be made transparent and presented to the public in a comprehensible way. This would be important not only because involving the public and securing its support is of key relevance for the future of this type of research, which may bring therapeutic benefits. But perhaps more importantly, because it keeps an open discussion around what it means to be human, or a person as well as about the sort of capacities and features we think instantiate moral status. 
Acknowledgments We would like to thank the Brocher Foundation for supporting the writing of this paper through their visiting fellowship program. We would also like to thank the insightful comments and suggestions of our blind reviewers.

\section{References}

Academy of Medical Sciences (2010). Exploring the Boundaries: Report on a public dialogue into animals containing human material.

Alzheimer's Disease International (2009). World Alzheimer's report.

Araki, R., Uda, M., Hoki, Y., Sunayama, M., Nakamura, M., Ando, S., et al. (2013). Negligible immunogenicity of terminally differentiated cells derived from induced pluripotent or embryonic stem cells. Nature, 494(7435), 100-104. doi:10.1038/nature11807.

Baker, M. (2013). Safety of induced stem cells gets a boost. Nature, 493(7431), 145. doi:10.1038/ 493145a.

Balaban, E., Teillet, M., \& Le Douarin, N. (1988). Application of the quail-chick chimera system to the study of brain development and behaviour. Science, 241(4871), 1339-1342. doi:10.1126/science. 3413496.

Barker, R. A. (2012). Stem cells and neurodegenerative diseases: where is it all going? Regenerative Medicine, 7(6s), 26-31. doi:10.2217/rme.12.64.

Baylis, F., \& Fenton, A. (2007). Chimera research and stem cell therapies for human neurodegenerative disorders. Cambridge Quarterly of Healthcare Ethics, 16(02), 195-208. doi:10.1017/ S0963180107070211.

Boland, D. F., \& Stacy, M. (2012). The economic and quality of life burden associated with Parkinson's disease: A focus on symptoms. The American Journal of Managed Care, 18(7 Suppl), S168-S175.

de Melo-Martin, I. (2009). Chimeras and Human dignity. Kennedy Institute of Ethics Journal, 18(4), 331-346.

Dunnett, S. B., \& Rosser, A. E. (2014). Challenges for taking primary and stem cells into clinical neurotransplantation trials for neurodegenerative disease. Neurobiology of Disease, 61, 79-89. doi:10.1016/j.nbd.2013.05.004.

Deutsher Ethikrat (2011). Human-animal mixtures in research. [Berlin: German Ethics Council (Opinion)].

Emborg, M. E., Liu, Y., Xi, J., Zhang, X., Yin, Y., Lu, J., et al. (2013). Induced pluripotent stem cellderived neural cells survive and mature in the nonhuman primate brain. Cell Reports, 3(3), 646-650. doi:10.1016/j.celrep.2013.02.016.

ESTOOLS. (2008). Ethical aspects of research on interspecies embryos and iPS cells. Sweden: Lund.

European Commission (2013). Neurodegenerative Disorders.

Fangerau, H., Fegert, J. M., \& Trapp, T. (2011). Implanted minds: The neuroethics of intracerebral stem cell transplantation and deep brain stimulation. Bielefeld: Transcript Verlag.

Greely, H. T., Cho, M. K., Hogle, L. F., \& Satz, D. M. (2007). Thinking about the human neuron mouse. The American Journal of Bioethics, 7(5), 27-40.

Greene, M. (2005). Human to non-human primate neural grafting: is it justified? http://www.ethikrat.org/ dateien/pdf/greene_h-nhp-grafting-motivations.pdf.

Greene, M., Schill, K., Takahashi, S., Bateman-House, A., Beauchamp, T., Bok, H., et al. (2005). Ethics: Moral issues of human-non-human primate neural grafting. Science, 309(5733), 385-386. doi:10. 1126/science. 1112207.

Grunwell, J., Illes, J., \& Karkazis, K. (2009). Advancing neuroregenerative medicine: A call for expanded collaboration between scientists and ethicists. Neuroethics, 2(1), 13-20. doi:10.1007/s12152-0089025-5.

Harding, J., Roberts, R. M., \& Mirochnitchenko, O. (2013). Large animal models for stem cell therapy. Stem Cell Research \& Therapy, 4(2), 23. doi:10.1186/scrt171.

Harvey, A., \& Salter, B. (2012). Anticipatory governance: Bioethical expertise for human/animal chimeras. Science as Culture, 21(3), 291-313.

Held, V. (2006). The ethics of care: Personal, political, and global. Oxford; New York: Oxford University Press.

Human Fertilisation and Embriology Authority (2007a). Hybrids and chimeras: a consultation on the ethical and social implications of creating human/animal embryos in research. London, UK. 
Human Fertilisation and Embryology Authority (2007b). Hybrids and chimera: A report on the findings of the consultation.

Hunther, C. (2009). Chimeras: The ethics of creating human-animal interspecifics. Munchen: Universitat Munchen, Ludwig-Maximilians-Universitat Munchen.

Hyun, I., Taylor, P., Testa, G., Dickens, B., Jung, K. W., McNab, A., et al. (2007). Ethical standards for human-to-animal chimera experiments in stem cell research. Cell Stem Cell, 1(2), 159-163. doi:10. 1016/j.stem.2007.07.015.

Karpowicz, P., Cohen, C. B., \& Van der Kooy, D. (2005). Developing human-nonhuman chimeras in human stem cell research: Ethical issues and boundaries. Kennedy Institute of Ethics Journal, 15(2), $107-134$.

Kobayashi, N. R. (2003). A scientist crossing a boundary: a step into the bioethical issues surrounding stem cell research. [Comment]. The American Journal of Bioethics, 3(3), W15-W16. doi:10.1162/ 15265160360706750.

Kriks, S., Shim, J. W., Piao, J., Ganat, Y. M., Wakeman, D. R., Xie, Z., et al. (2011). Dopamine neurons derived from human ES cells efficiently engraft in animal models of Parkinson's disease. Nature, 480(7378), 547-551. doi:10.1038/nature10648.

Lindvall, O., Rehncrona, S., Gustavii, B., Brundin, P., Astedt, B., Widner, H., et al. (1988). Fetal dopamine-rich mesencephalic grafts in Parkinson's disease. Lancet, 2(8626-8627), 1483-1484.

MacKellar, C. (2013). Differentiating between human and non-human interspecies embryos. Journal of Medical Ethics,. doi:10.1136/medethics-2013-101363.

Macrì, S., Ceci, C., Altabella, L., Canese, R., \& Laviola, G. (2013). The Directive 2010/63/EU on animal experimentation may skew the conclusions of pharmacological and behavioural studies. Science Report 3. doi:10.1038/srep02380.

Magnani, T. A. (1999). The patentability of human-animal chimeras. Berkeley Tech. L.J., 14, 443-460.

Menache, A. (2010). The replacement of human primates in brain research. Strasbourg: One Voice (Report).

NEK (2006). Research involving human embryos and fetuses. [Bern: National Ethikkommission im Bereich Humanmedizin (NEK) (Opinion no. 11/2006)].

Nuffield Council on Bioethics (2005). The ethics of research involving animals. London, UK.

Park, I. H., Arora, N., Huo, H., Maherali, N., Ahfeldt, T., Shimamura, A., et al. (2008). Disease-specific induced pluripotent stem cells. Cell, 134(5), 877-886. doi:10.1016/j.cell.2008.07.041.

Pluchino, S., Gritti, A., Blezer, E., Amadio, S., Brambilla, E., Borsellino, G., et al. (2009). Human neural stem cells ameliorate autoimmune encephalomyelitis in non-human primates. Annals of Neurology, 66(3), 343-354. doi:10.1002/ana.21745.

Purves, D., Augustine, G., Fitzpatrick, D., \& Al, E. (2001). Development of language: a critical period in humans. In D. Purves, G. Augustine, D. Fitzpatrick, \& E. Al (Eds.), Neuroscience (2nd ed.). Sunderland (MA): Sinauer Asscociated.

Redmond, D. E., Bjugstad, K. B., Teng, Y. D., Ourednik, V., Ourednik, J., Wakeman, D. R., et al. (2007). Behavioral improvement in a primate Parkinson's model is associated with multiple homeostatic effects of human neural stem cells. Proceedings of the National Academy of Sciences, 104(29), 12175-12180. doi:10.1073/pnas.0704091104.

Reusser, R., \& Schweizer, R. J. (2008). Kommentar zur Art. 119. In B. Ehrenzeller, P. Mastronardi, R. J. Schweizer, \& K. A. Vallender (Eds.), Die schweizerische Bundesverfassung- Kommentar, 2. Auflage, dike Verlag und Schulthess Juristische Medien (St Gallen Commentary on the Federal Constitution).

Ricceri, L., \& Vitale, A. (2011). The law through the eye of a needle. EMBO Report, 12(7), 637-640.

Robert, J. S. (2006). The science and ethics of making part-human animals in stem cell biology. The FASEB Journal, 20(7), 838-845. doi:10.1096/fj.05-42861sf.

Robert, J. S., \& Baylis, F. (2003). Crossing species boundaries. The American Journal of Bioethics, 3(3), 1-13. doi:10.1162/15265160360706417.

Rose, N. (2009). The Politics of Life Itself: Biomedicine, power, and subjectivity in the twenty-first century. Princeton: Princeton University Press.

Russell, W. M. S., \& Burch, R. L. (1959). The principles of humane experimental technique. London: Methuen.

Secretary of State for the Home Department (1986). Guidance on the Operation of the Animals (Scientific Procedures) Act 1986. UK.

Streiffer, R. (2005). At the edge of humanity: human stem cells, chimeras, and moral status. Kennedy Institute of Ethics Journal, 15(4), 347-370. 
Streiffer, R. (2010). Chimeras, moral status, and public policy: Implications of the abortion debate for public policy on human/nonhuman chimera research. The Journal of Law, Medicine \& Ethics, 38(2), 238-250. doi:10.1111/j.1748-720X.2010.00484.x.

Suran, M., \& Wolinsky, H. (2009). The end of monkey research? EMBO Report, 10(10), 1080-1082.

Swiss Academy of Medical Sciences (2009). Interspecies Crosses: Aspects of Animal Protection. [Basel: Ethics Committee for Animal Studies of the Swiss Academy of Medical Sciences and Swiss Academy of Sciences (Position statement)].

Swiss Academies of Arts and Sciences (2010). The dignity of animals and the evaluation of interests in the Swiss Animal Protection Act. [Ethics Committee for Animal Experimentation of the Swiss Academies of Arts and Sciences (Position statement)].

Tamaki, S., Eckert, K., He, D., Sutton, R., Doshe, M., Jain, G., et al. (2002). Engraftment of sorted/ expanded human central nervous system stem cells from fetal brain. Journal of Neuroscience Research, 69(6), 976-986. doi:10.1002/jnr.10412.

The Academy of Medical Sciences (2011). Animals containing human material.

The Danish Council of Ethics (2008). Man or Mouse? Ethical aspects of chimera research.

The National Academies. (2005). Guidelines for human embryonic stem cell research. Washington: The National Academies Press.

Tomasello, M., \& Call, J. (2011). Methodological challenges in the study of primate cognition. Science, 6060, 1227-1228. doi:10.1126/science.1213443.

Uchida, N., Buck, D. W., He, D., Reitsma, M. J., Masek, M., Phan, T. V., et al. (2000). Direct isolation of human central nervous system stem cells. Proceedings of the National Academy of Sciences, 97(26), 14720-14725. doi:10.1073/pnas.97.26.14720.

Vierbuchen, T., Ostermeier, A., Pang, Z. P., Kokubu, Y., Sudhof, T. C., \& Wernig, M. (2010). Direct conversion of fibroblasts to functional neurons by defined factors. Nature, 463(7284), 1035-1041. doi: $10.1038 /$ nature 08797.

Walter, S. (2010). Locked-in syndrome, BCI, and a confusion about embodied, embedded, extended, and enacted cognition. Neuroethics, 3(1), 61-72. doi:10.1007/s12152-009-9050-z.

World Health Organization (2003). Global Declaration for Parkinson's Disease. 